\title{
Dynamic Financial Risk Management
}

\author{
December 8, 2005
}

\section{Pauline Barrieu ${ }^{1}$ and Nicole El Karoui ${ }^{2}$}

\section{Introduction}

For many years, financial markets have been part of people's everyday life. Information on the major securities can be found easily in the daily press or on internet. The different actors of the economy look closely at some fundamental parameters, such as interest rates, currency rates, stock prices..., which may affect the management of their firms, especially after the deregulation process initiated in the United States in the 1970s. The accrued international dimension and efficiency of the communication means have extended this phenomenon to the entire world economy. Small investors savings and pensions are also exposed to the risks generated by variations in these factors.

Financial institutions are aware of the importance of managing these risks. They offer different solutions, which will be presented below. For instance, the following product can help small investor to deal with variations of the stock markets:

In 2005, bank XYZ offered the following product, which is a 5 years guaranteed capital fund:

- $0 \%$ of the yield of a basket of six indices when it is negative.

- $50 \%$ of the yield if lower than $50 \%$

- $25 \%+100 \%$ of the yield if greater than $50 \%$

The basket is build with six financial indices from different international markets: FTSE 89, SMI, FTSE100, NIKKEI225, SEP500, Hang Seng.

More generally, in order to deal with variations in fundamental financial and economic factors, a large variety of financial instruments has been created. Simple derivative contracts (futures, options, swaps, etc.) or more exotic financial products (credit derivatives, catastrophe bonds, exotic options, etc.) are offered to private and institutional investors to transfer their financial risks to specialized financial institutions in exchange of a suitable compensation. The organized markets, on which these products are traded, are fully devoted to the management of (fundamental) financial risks. They can be seen as financial risk markets. As argued by Merton [36], the development of the financial risk industry would not have been possible without the development and support of theoretical tools. Mathematics has emerged as the leading discipline to address fundamental questions related to financial risk management. Mathematical finance, which applies the theory of probability and stochastic processes to finance, in particular Brownian motion and martingale theory, stochastic control and partial differential equations, is now a field of research in its own.

\footnotetext{
${ }^{1}$ L.S.E., Statistics Department, Houghton Street, London, WC2A 2AE, United Kingdom. (e-mail: p.m.barrieu@lse.ac.uk)

${ }^{2}$ C.M.A.P., Ecole Polytechnique, 91128 Palaiseau Cédex, France. (e-mail: elkaroui@cmapx.polytechnique.fr)
} 


\section{The Black-Scholes-Merton paradigm of zero-risk}

The trigger for the whole financial industry expansion has been surprisingly "the Brownian motion theory and Itô stochastic calculus", first introduced in finance by Bachelier in this PhD thesis in 1900 [4], then used again by Black, Scholes and Merton in 1973 [6].

In particular, they have provided a method to price and hedge derivative instruments, which are financial contracts delivering a payoff $H(\omega)$, depending upon the scenario $\omega$ at a maturity date. The typical example is the (European) call option, which offers a protection in case of a large increase in the underlying asset price. More precisely, a European call provides its buyer with the right (and not the obligation) to purchase the risky asset at a pre-specified price (the exercise price) $K$ at a pre-specified date $T$ in the future (maturity date). The potential gain at maturity can therefore be written as $\left(X_{T}-K\right)^{+}$, where $X_{T}$ denotes the value of the underlying asset at maturity.

Note that the product described in the introduction is in fact a combination of such options, based on a basket of six indices.

Black, Scholes and Merton have developed the completely new idea according to which it is possible for an option seller to deliver the contract at maturity without incurring any residual risk by using a dynamic trading strategy on the underlying asset. The stochastic arguments may discourage many people; it is however possible to reduce technical difficulties, and to develop arguments which are essentially probability-free, as first introduced by Foellmer [23].

\section{A dynamic uncertain world}

The uncertainty is modeled via a family $\Omega$ of scenarios $\omega$, characterizing the possible trajectories of the asset prices in the future. Such paths are described as positive continuous function $\omega$ with coordinates $X_{t}=\omega(t)$, such that the continuous quadratic variation exists: $[X]_{t}(\omega)=\lim _{n} \sum_{t_{i} \leq t, t_{i} \in D_{n}}\left(X_{t_{i+1}}-X_{t_{i}}\right)^{2}$ along the sequence of dyadics partitions $D_{n}$.

The pathwise version of stochastic calculus yields to Itô's formula

$$
\begin{aligned}
f\left(t, X_{t}\right)(\omega) & =f\left(0, x_{0}\right)+\int_{0}^{t} f_{x}^{\prime}\left(s, X_{s}\right)(\omega) d X_{s}(\omega)+\int_{0}^{t} f_{t}\left(s, X_{s}\right)(\omega) d t \\
& +\int_{0}^{t} \frac{1}{2} f_{x x}^{\prime \prime}\left(s, X_{s}\right)(\omega) d[X]_{s}(\omega)
\end{aligned}
$$

The last two integrals are well defined as Lebesgue-Stieljes integrals, while the first exists as an Itô's integral, defined as the limit of non-anticipating Riemann sums $\sum_{t_{i} \leq t, t_{i} \in D_{n}} \delta_{t_{i}}(\omega)\left(X_{t_{i+1}}-X_{t_{i}}\right)$, where $\delta_{t}=f_{x}^{\prime}\left(t, X_{t}\right)$.

This formulation has a direct financial interpretation. More precisely, the Itô's integral may be seen as the cumulative gain process of a trading strategy where $\delta_{t}$ is the number of the shares of the underlying asset held at time $t$ and the increment in the Riemann sum is the price variation over the period. The non-anticipating assumption corresponds to the financial requirement that the investment decisions are based only on the observation of the past prices. At any time $t$, the residual wealth of the trader is invested in cash, yielding a rate (called short rate) $r_{t}$ by time unit.

A natural condition is imposed on the strategy, forcing the associated portfolio to be self-financing. In other words, no money is added to or removed from the portfolio after its construction at time 0 . This is expressed as:

$$
d V_{t}=r_{t}\left(V_{t}-\delta_{t} \cdot X_{t}\right) d t+\delta_{t} \cdot d X_{t}=r_{t} V_{t} d t+\delta_{t} \cdot\left(d X_{t}-r_{t} X_{t} d t\right), V_{0}=z
$$

\section{Hedging derivatives: solving a target problem}

We now come back to the problem of a trader having to pay at maturity $T$ the amount $h\left(X_{T}\right)(\omega)$ in the scenario 
$\omega\left(h\left(X_{T}\right)(\omega)=\left(X_{T}(\omega)-K\right)^{+}\right.$for a European call option). The trader wants to hedge his position in the sense that this target payoff is replicated by the wealth generated by a self-financing portfolio in all scenarios at maturity. The "miraculous" message in the world of Black and Scholes is that such a perfect hedge is possible and easily computable.

The additional assumption is that the quadratic variation is absolutely continuous $d[X]_{t}=\sigma\left(t, X_{t}\right) X_{t}^{2} d t$. The (regular) strictly positive function $\sigma(t, x)$ is a key parameter in financial markets, called the local volatility.

Looking for the wealth at any time $t$ as a function $f\left(t, X_{t}\right)$, we see that, given (1) and (2),

$$
\begin{aligned}
d f\left(t, X_{t}\right) & =f_{t}^{\prime}\left(t, X_{t}\right) d t+f_{x}^{\prime}\left(t, X_{t}\right) d X_{t}+\frac{1}{2} f_{x x}^{\prime \prime}\left(t, X_{t}\right) X_{t}^{2} \sigma^{2}\left(t, X_{t}\right) d t \\
& =f\left(t, X_{t}\right) r_{t} d t+\delta\left(t, X_{t}\right)\left(d X_{t}-X_{t} r_{t} d t\right)
\end{aligned}
$$

By identifying the $d X_{t}$ terms (thanks to the assumption $\sigma(t, x)>0$ ), we see that $\delta\left(t, X_{t}\right)=f_{x}^{\prime}\left(t, X_{t}\right)$, and that $f$ should be solution of the following partial differential equation, called Pricing PDE:

$$
f_{t}^{\prime}(t, x)+\frac{1}{2} f_{x x}^{\prime \prime}(t, x) x^{2} \sigma^{2}(t, x)+f_{x}^{\prime}(t, x) x r_{t}-f(t, x) r_{t}=0, f(T, x)=h(x)
$$

The derivative price at time $t_{0}$ must be $f\left(t_{0}, x_{0}\right)$, if not, it is easy to generated profit without bearing any risk, which is an arbitrage opportunity. This is the rule of the unique price, which holds true in a liquid market.

Note that the volatility of the asset return is the only parameter of the underlying dynamics appearing in the PDE. The instantaneous return does not play any role, only the size of the fluctuations matters, not the direction. The fact that the option price does not depend on the market trend is a fundamental result. It could first seem surprising, as the main motivation of this financial product is to hedge the purchaser against underlying rises. But the possibility to replicate the option by a dynamic hedging strategy makes the framework risk-neutral and de-trend the market.

The PDE's fundamental solution $q(t, x, T, y)\left(h(x)=\delta_{y}(x)\right)$ may be reinterpreted in terms of the Arrow-Debreu "states prices" density, introduced in 1953 by this Nobel Price winner, with a purely theoretical economical point of view and by completely different arguments.

The pricing rule of the derivative contract becomes: $f(t, x)=\int h(y) q(t, x, T, y) d y$, where $q$ is the pricing kernel. When $\sigma(t, x)=\sigma_{t}$ is a deterministic function, the pricing kernel is the log-normal density, deduced from the Gaussian distribution by an explicit change of variable. The closed-form formula ${ }^{3}$ for European call option price is the famous Black and Scholes formula ${ }^{4}$. The impact of this methodology was so important that Black, Scholes and Merton received the Nobel prize for economics in 1997.

\subsection{From Market Risk to Model Risk}

In practice, even in the simple Black-Scholes framework presented above (denoted by B\&S in the following), a key problem is to calibrate the model parameters (in particular the volatility), the dynamics of which is given

\footnotetext{
${ }^{3}$ Obtaining closed-form formulae has a certain advantage, even if it is for very simple (over-simple) models, as they can be used as test functions for numerical procedure.

${ }^{4}$ In the Black-Scholes model with constant coefficients, the Call option price $C^{B S}(t, x, K, T)$ is given via the Gaussian cumulative function $\mathcal{N}(z)=\int_{\infty}^{z} \frac{1}{\sqrt{2 \pi}} e^{-\frac{y^{2}}{2}} d y$, and $\theta=T-t$,

$$
\left\{\begin{array}{c}
C^{B S}(t, x, t+\theta, K)=x \mathcal{N}\left(d_{1}(\theta, x / K)\right)-K e^{-r \theta} \mathcal{N}\left(d_{0}(\theta, x / K)\right) \\
d_{0}(\theta, x / K)=\frac{1}{\sigma \sqrt{\theta}} \log \left(\frac{x}{K e^{-r \theta}}\right)-\frac{1}{2} \sigma \sqrt{\theta}, \quad d_{1}(\theta, x / K)=d_{0}(\theta, x / K)+\sigma \sqrt{\theta}
\end{array}\right.
$$

Moreover $\Delta(t, x)=\partial_{x} C^{B S}(t, x, t+\theta, K)=\mathcal{N}\left(d_{1}(\theta, x / K)\right)$.
} 
by the following stochastic differential equation (SDE):

$$
d X_{t}=X_{t}\left(\mu\left(t, X_{t}\right) d t+\sigma\left(t, X_{t}\right) d W_{t}\right), X_{t_{0}}=x_{0}
$$

The Brownian motion $W$ may be viewed as a standardized Gaussian noise with independent increments. The instantaneous return $\mu\left(t, X_{t}\right)$ is a trend parameter, that will not play any role in the derivative pricing as noticed in the previous section.

\subsubsection{Statistical versus implied point of view}

In the $\mathrm{B} \& \mathrm{~S}$ model with constant parameter, the square of the volatility is the variance by time unit of the $\log$ return $R_{t}=\ln \left(X_{t}\right)-\ln \left(X_{t-h}\right)$. If the only available information is the asset price historical data, the statistical estimator to be used is the empirical variance, computed over a more or less long time period [0,N] as $\widehat{\sigma}^{2}=\frac{1}{N-1} \sum_{i=0}^{N-1}\left(R_{t_{i}}-\overline{R_{N}}\right)^{2}$ where $\overline{R_{N}}=\frac{1}{N} \sum_{i=0}^{N-1} R_{t_{i}}$. This estimator is called historical volatility 5 . Such an approach is very natural, especially in certain new markets based on energy or on weather.

However, traders use this estimator with some reserve. They argue indeed that financial markets are not "statistically" stationary and that past is not enough to explain the future.

When possible, traders use the additional information given by the quoted option prices, $C^{\text {obs }}(T, K)$ for different maturities $T$ and different strike prices $K$, and convert it into volatility parametrization. The implied volatility they obtain is implicitly defined as: $C^{o b s}(T, K)=C^{B S}\left(t_{0}, x_{0}, T, K, \sigma^{i m p}\right)$. Moreover, the option hedge portfolio is easily computed by $\Delta^{i m p}=\partial_{x} C^{B S}\left(t_{0}, x_{0}, T, K, \sigma^{i m p}\right)$. This strategy is used dynamically by defining the implied volatility and the associated $\Delta$ at any renegotiation dates. This specific use of the Black \& Scholes formula based on the hedging strategy may explain its success.

The option real world is not Black \& Scholes This attractive methodology has rapidly appeared to be limited: observed implied volatilities are depending on the option parameters (time to maturity and exercise price). This implied volatility surface is in complete contradiction with the assumptions of the B\&S model. In particular, the market quotes large movements, resulting in heavy tail distributions, higher than in the lognormal framework.

In order to take into account this empirical observation, the first ideas has been to consider a model with local volatility $\sigma(t, x)$. In 1995, Dupire [18] related the implied volatility with the first and second partial derivatives of the option price by giving a clever formulation for the dual PDE (one dimensional in state variable). More precisely, when the market conditions are $\left(t_{0}, x_{0}\right)$ and for the sake of simplicity the short rate is assumed to be null, then

$$
C_{T}^{\prime}(T, K)=\frac{1}{2} \sigma^{2}(T, K) K^{2} C_{K K}^{\prime \prime}(T, K), \quad C\left(t_{0}, x_{0}\right)=\left(x_{0}-K\right)^{+} .
$$

where $C(T, K)$ is the price of the call option with parameters $(T, K)$.

This formula is especially attractive as it gives a simple relation between "quoted Call option prices". The local volatility is computable from a continuum of coherent (without arbitrage) observed quoted prices as $\sigma^{2}(K, T)=2 \frac{C_{T}^{\prime}(T, K)}{K^{2} C_{K K}^{\prime \prime}(T, K)}$.

\subsubsection{Ill-posed inverse problem for PDE}

Unfortunately, the option market is typically limited to a relatively few different exercise prices and maturities; therefore, a naive interpolation yields to some irregularity and instability of the local volatility. The problem of determining local volatility can be viewed as a non-linear problem of function approximation from a finite data set. This data set is the value $C_{i, j}$ of the solution at $\left(t_{0}, x_{0}\right)$ of PDE (3) with boundary conditions

\footnotetext{
${ }^{5}$ We do not speak about the increasing research interest in statistical financial modeling as it is a subject in itself.
} 
$h_{i, j}(x)=\left(x-K_{i, j}\right)^{+}$at maturity $T_{i}$. Setting the problem as a PDE's inverse problem yields to more robust calibration methods. These ideas appear for the first time in finance in 1995 [32], but the problem is not standard because of the strongly non-linear relationship existing between option prices and local volatility; moreover, the considered data set is related to a single initial condition. Prices adjustment is made through a least square minimization program, including a penalization term related to the local volatility regularity. Obtaining a numerical solution of this complex problem is extremely time consuming.

\subsubsection{Model risk}

In this local volatility problem, for instance, we may end up with several possible solutions depending on the selection criterion, in particular on the penalization that is used. Therefore, beyond the calibration problem there is a more fundamental issue related to the choice of a given model for the underlying dynamics. Choosing a particular model has an impact on the derivative prices and on the hedging strategy the trader obtains and therefore on the risk he undertakes.

The market risk itself appears to be not so important in the sense that it can be hedged (even partially) by using derivative instruments and replicating portfolios. Measuring the model risk is very difficult and barely taken into account by the agents in the market. This risk becomes all the more important so since some financial risks cannot be hedged directly, as the volatility risk. To find the price of a derivative, the key argument of replication on which the $\mathrm{B} \& \mathrm{~S}$ model is based, cannot be used any longer, as we will see in the next section.

\section{Super-replication and Risk Measures}

The fundamental assumption of the B\&S theory is the options market may be entirely explained by underlying prices. In economic theory, it corresponds to a situation of market efficiency: a security price contains all the information relative to this particular security.

In an option world, the observed statistical memory of historical volatility leads naturally to consider stochastic volatility models. More precisely, let assume that the volatility parameter $\sigma$ of the asset price $X$ has now its own specific risk, represented by a stochastic factor $Y$ with $d Y_{t}=\eta\left(t, X_{t}, Y_{t}\right)+\gamma\left(t, X_{t}, Y_{t}\right) d \tilde{W}_{t}$ where $\tilde{W}$ is a Brownian motion, which might be correlated with the Brownian motion driving $X$. What does it change? In fact, everything! Perfect replication by a portfolio is not possible any more; the notion of unique price does not exist any longer... But, such a situation, which appears to be non-standard, is often the general case. What kind of answer can we bring to such a problem?

\subsection{Super-replication and Robust Hedging}

The option problem is still a target problem $C_{T}$, to be replicated by a portfolio $V_{T}(\pi, \delta)=\pi+\int_{0}^{T} \delta_{s} d X_{t}$ depending on market asset $X$. The investment decision $\left(\delta_{t}\right)$ is taken from available information at time $\mathrm{t}$ (in an adapted way) ${ }^{6}$. Some other constraints (size, sign...) may also be imposed on $\delta$. Let $\mathcal{V}_{T}$ be the set of all derivatives, that can be replicated at time $T$ by an admissible portfolio. Their price at $t_{0}$ is given by the value of their replicating portfolio.

Super-replicating $C_{T}$ is equivalent to find the smallest derivative $\widehat{C}_{T} \in \mathcal{V}_{T}$ which is greater than $C_{T}$ in all scenarios. The super-replication price is the price of such a derivative. The $\widehat{C}_{T}$ replicating portfolio is the $C_{T}$ robust hedging.

There are several ways to characterize the super-replicating portfolio:

\footnotetext{
${ }^{6}$ For the sake of simplicity, interest rates are assumed to be null and only one risky asset is traded on the market.
} 
1) Dynamic programming on level sets: this is the most direct (but least recent) approach. This method proposed by Soner and Touzi [39] has led the way for original works in geometry by giving a stochastic representation of a class of mean curvature-type geometric equations.

2) Duality: this second approach is based on a characterization of the "orthogonal space" of $\mathcal{V}_{T}$ in terms of martingale measures in $\mathcal{Q}_{T}$, that is probability measures such that the expectation of the $t$-time value of non-negative gain portfolios is equal to 0 . The super-replication price is then obtained as

$$
\widehat{C}_{0}=\sup _{Q \in \mathcal{Q}_{T}} \mathbf{E}_{Q}\left[C_{T}\right] .
$$

Super-replication prices are often too expensive to be used in practice. However, they give an upper bound to the set of possible prices.

We will develop the dual approach in Subsection 3.3, when looking at risk measures. The super replication price is indeed is very similar to the notion of convex risk measures, where the worst case is also considered.

\subsection{Martingale measures}

The idea of introducing a dual theory based on probability measures is first due to Bachelier [4] in 1900, even if it has been essentially developed by Harisson and Pliska [29]. The actual and most achieved form is due to Delbaen and Schachermayer [17] and to the very active group in theoretical mathematical finance.

More precisely, a martingale measure, $\mathbf{Q}$, is a probability measure characterized by: $\forall V_{T} \in \mathcal{V}_{T}, \mathbf{E}_{\mathbf{Q}}\left[V_{T}\right]=V_{0}$. When the set of admissible portfolios contains all simple strategies (the portfolio is renegotiated at discrete times, randomly chosen), the price of the fundamental asset $\left(X_{t}\right)$ is a $\mathbf{Q}$-(local) martingale, in the sense that the best estimation under $\mathbf{Q}$ of $X_{t+h}$ given the available information at time $t$ is $X_{t}$. Therefore, the financial game is fair with respect to martingale measures.

\section{Complete market}

When $\mathcal{V}_{T}$ contains all possible (path-dependent) derivatives, the market is said to be complete, and the set of martingale measures is reduced to a unique element $\mathbf{Q}$, often called risk-neutral probability. This is the case in the B\&S framework. The security dynamics becomes $d X_{t}=X_{t} \sigma\left(t, X_{t}\right) d W_{t}^{\mathbf{Q}}$ where $W^{\mathbf{Q}}$ is a $\mathbf{Q}$ Brownian motion. This formalism is really efficient as it leads to the following pricing rule: $\widehat{C}_{0}=\mathbf{E}_{\mathbf{Q}}\left(C_{T}\right)$. The computation of the option replicating portfolio is more complex. In the diffusion case, the price is function of risk factors and the replicating portfolio only depends on price partial derivatives.

\section{Incomplete market}

A market is said to be incomplete in any other situations. The characterization of the set $\mathcal{Q}_{T}$ is all the more delicate so since there are many different situations which may lead to market imperfections (non-tradable risks, transaction costs..). The abstract theory of super-replication (and more generally, of portfolio optimization under constraints) based on duality has been intensively developed; the super-replicating price process, described in Subsection 3.1 is proved to be a supermartingale with respect to any admissible martingale measures (see El Karoui and Quenez [19] or Kramkov [31]).

\subsection{Risk measures}

After having considered the trader's problem of pricing and management of his risk (hedging or super-hedging), we now adopt the point of view of the risk manager himself, or more generally that of the regulator, who both have to deal with partially hedged positions most of the time. Indeed, when the perfect replication is not possible, is too expensive, or when the model risk is too important, the trader has to measure his remaining market risk exposure. The traditional measure is the variance of the replicating error.

The regulator has a more global perspective and the risks he has to manage are those of the bank it-self, i.e. 
the sum of all the remaining risk exposures of the different traders. The safety and perenniality of the bank depends on his measure of this global risk. Therefore, the variance appears to be over-simplistic, as it does not really focus on the downside part of the risk distribution. New criteria taking into account extreme events, have been imposed, transforming the risk management at both quantitative and qualitative levels.

The Value at Risk (VaR) criterion, corresponding to the maximal level of losses acceptable with a given probability (usually 95\%), has taken a considerable importance for several years. In 1995, the Basel Committee on Banking Supervision imposed market risk capital requirements for all financial institutions. These are based upon a daily VaR computation of the global risky portfolio. Such an assessment is essential from the operational point of view, as it impacts the provisions a bank has to hold to face market risks.

A huge debate has been however initiated by the academic world [1] on the VaR significance and consistency as a risk measure. In particular, its non-additive property, incompatible with the key idea of diversification, enables banks to play with subsidiary creations. This debate has received an important echo from the professional world, which is possibly planning to review this risk measure criterion. Sub-additive and coherent risk measures are an average estimation of losses with respect to a family of probability measures $\mathcal{Q}_{\rho}$ as $\rho(X)=\sup _{Q \in \mathcal{Q}_{\rho}} \mathbf{E}_{Q}(-X)$. This characterization has been extended to convex risk measures [27], by adding a penalization term on probability density, a typical example being the entropy.

The set of probability measures $\mathcal{Q}_{\rho}$ of the risk measure dual representation could describe different scenario anticipations or beliefs of the risk manager. The underlying framework is not necessarily dynamic, as it was previously when studying the dynamic hedging problem. But the set $\mathcal{Q}_{\rho}$ can be also closer to a dynamic market view in the sense that certain financial instruments have to be risk-free under these measures. In this latter case, the risk measure is very similar to a super-replication price (from the seller point of view), previously described. Even if the motivations of the risk manager and of the trader are a priori completely different, they end up with the same assessment of their risk, as soon as no perfect hedge is possible and some unhedgeable risks remain.

\section{A new research field: Securitization}

As described by Cummins in [14], "securitization is one of the most important innovations of modern finance. The securitization process involves the isolation of a pool of assets or rights to a set of cash flows and the repackaging of the assets or cash flows into securities that are traded in capital markets. The trading of cash flow streams enables the different parties to the contract to manage and diversify their risk, to take advantages of arbitrage opportunities or to invest in new classes of risk that enhance market efficiency."

\subsection{New products}

More precisely, the first securitization of bank loans took place in the 1970s in the United States. Several banks pooled their mortgages together, repackaged them and sold them on financial markets, creating a new type of securities, called Mortgage-Backed Securities. Since then, this securitization process has been generalized to other banking activities. Some complex structures have been recently issued on the credit risk market: the famous CDOs (Collateralized Debt Obligations), consisting of the repackaging of cash flows subject to credit risk, i.e. risk of default of the counterpart. By this securitization process, the individual risk is reduced through diversification. This creates however a new risk related to the correlation and joint default of the different counterparts of the banks.

From a modeling point of view, risks have been represented in a continuous way so far. Now, modeling potential losses dynamically and allowing assets to default require the introduction of some processes with jumps. This 
possibility of default makes the financial market incomplete, the default being typically unhedgeable. Using all the theoretical framework developed previously in a continuous framework, new approaches of the dynamic hedging problem have to be adopted take into account the new discontinuities.

\subsection{New perspectives at the interface between finance and insurance}

The past decade has seen the emergence of a range of financial instruments depending on risks traditionally considered to be within the remit of the insurance sector. Examples are weather and catastrophe claims, contingent on the occurrence of certain weather or catastrophic events. The development of instruments at the interface of insurance and finance raises new questions, not only about their classification but also about their design, pricing and management. The pricing issue is particularly intriguing as it questions the very logic of such contracts. Indeed, standard principles for derivatives pricing based on replication do not apply any more because of the special nature of the underlying risk. On the other hand, the question of the product design, unusual in finance, is raised since the logic behind these products is closer to that of an insurance policy (see for instance Barrieu and El Karoui [5]).

\subsection{Problems related to the dimension}

A key problem associated with the development of these products is the repackaging of the cash flows themselves just as the price. The high-dimension of the problem makes these questions even harder. Several techniques have been recently developed to tackle this issue.

Identifying the different risk directions and the impact of estimation methods (for instance, when computing the VaR, the number of observations is less than that of risk factors...) is crucial not only to study a bank portfolio VaR, but also to hedge derivatives depending on a large number of underlying assets. Random matrix theory or other asymptotic tools may bring some new ideas on this question.

Finally, by presenting the most important tools of the financial risk industry, we have voluntary left apart anything on financial asset statistical modeling, which may be the subject of a whole paper on its own. It is clear that the VaR criterion, the market imperfections are highly dependent on an accurate analysis of the real and historical world. Intense and very innovating research is now developed on related topics (High-frequency data, ARCH and GARCH processes, Lévy processes with long memory, random cascades).

\section{Conclusion}

As a conclusion, applied mathematicians have been highly questioned by problems coming from the financial risk industry. This is a very active world, rapidly evolving, in which theoretical thoughts often have immediate and practical impacts and applications. Moreover, practical constraints generate new theoretical problems. This presentation is far from being an exhaustive view of the financial problems. Many exciting problems, from both theoretical and practical points of view, have not been presented.

\section{References}

[1] P.Artzner, F.Delbaen, J.M.Eber and D.Heath (1999) Coherent measures of risk. Mathematical Finance Vol. 9, no. 3,pp. 203-228.

[2] M. Avellaneda (1998) The minimum-entropy algorithm and related methods for calibrating asset-pricing model. Trois applications des mathématiques. SMF Journ. Annu. 1998, pp. 51-86. 
[3] M. Avellaneda, C. Friedman, R. Holmes and D. Samperi (1997) Calibrating volatility surfaces via relative entropy minimization. Applied Mathematical Finance, Vol. 4, pp. 37-64.

[4] L. Bachelier (1900) Théorie de la spéculation. Annales scientifiques de l'Ecole Normale Supérieure, III-17, pp. 21-86. Reproduit par les Editions Jacques Gabay en 1995.

[5] P. Barrieu, N. El Karoui (2005) Inf-convolution of Risk Measures and Optimal Risk Transfer. Finance and Stochastics, vol. 9, pp. 269-298.

[6] F. Black and M.Scholes (1973) The Pricing of Options and Corporate Liabilities. Journal of Political Economy, Vol. 72, pp. 637-659.

[7] H. Berestycki, J. Busca and I. Florent (2002) Asymptotics and calibration of local volatility models. Quantitative Finance, Vol. 2, pp. 61-69.

[8] B. Bouchard and N. Touzi (2004) On the Malliavin approach to Monte Carlo approximation of conditional expectations. Finance and Stochastics, Vol. 8, pp. 45-71.

[9] J.P. Bouchaud, D. Sornette, C. Walter and J.P. Aguilar (1998) Taming large events : Optimal portfolio theory for strongly fluctuating assets. International Journal of Theoretical and Applied Finance, Vol. 1 no 1, pp. 25-41.

[10] I. Bouchouev and V. Isakov (1999) Uniqueness and stability for inverse problems that arise in financial markets. Inverse Problems, pp. 95-116.

[11] M. Broadie, P. Glasserman, Z. Ha (2000) Pricing American Options by Simulation Using a Stochastic Mesh with Optimized Weights. Probabilistic Constrained Optimization: Methodology and Applications, S. Uryasev, ed., Kluwer, pp. 32-50.

[12] T. Coleman, Y. Li and A. Verma (1999) Reconstructing the unknown volatility function. Journal of Computational Finance, Vol. 2, no 3, pp. 77-102.

[13] R. Cont and J. Da Fonseca (2001) Deformation of implied volatility surfaces : an empirical approach. Empirical Finance, Takayasu (Ed.), Tokyo: Springer.

[14] D. Cummins (2004) Securitization of Life Insurance Assets and Liabilities. Presentation at the 31st Seminar of the European Group of Risk and Insurance Economics.

[15] J. Cvitanić and I. Karatzas (1999) On dynamic measures of risk. Finance and Stochastics, Vol. 3, no. 4, pp. 451-482.

[16] M. Davis (2001) Pricing weather derivatives by marginal value. Quantitative Finance, Vol. 1, pp. 1-4.

[17] F. Delbaen and W. Schachermayer (1994) A General Version of the Fundamental Theorem of Asset Pricing. Mathematische Annalen, Vol. 300, pp. 463-520.

[18] B. Dupire (1997) Pricing and hedging with smiles. Mathematics of derivative securities. Dempster and Pliska eds., Cambridge Uni. Press, pp. 103-112.

[19] N. El Karoui and M.C. Quenez (1995) Dynamic programming and pricing of contigent claims in incomplete markets. SIAM Journal of Control and Optimization, Vol. 33, no 1, pp. 29-66. 
[20] N. El Karoui, S. Peng and M.C. Quenez (1997) Backward stochastic differential equations in finance. Mathematical Finance, Vol. 7, no. 1, pp. 1-71.

[21] P. Embrechts, A. Hoeing and A. Juri (2003) Using Copulae to bound the Value-at-Risk for functions of dependent risk. Finance and Stochastics. Vol. 7, pp. 145-167.

[22] P. Embrechts, C. Klueppelberg and T. Mikosch (1997) Modelling Extremal Events for Insurance and Finance. Springer book.

[23] H. Foellmer (2001) Probabilistic aspects of financial risk. Proceedings of the European Congress of Mathematics, Barcelona 2000. Birkhauser.

[24] H. Foellmer and D.O. Kramkov (1997) Optional decompositions under constraints. Probability Theory and Related Fields, Vol. 109, pp. 1-25.

[25] M. Frittelli (2000) The minimal entropy martingale measure and the valuation in incomplete markets. Mathematical Finance, Vol. 10, no 1, pp. 39-52.

[26] H. Foellmer and P. Leukert (1999) Quantile hedging. Finance and Stochastics, Vol. 3, no. 3, pp. 251-273.

[27] H. Foellmer and M. Schied (2002) A Convex measures of risk and trading constraints. Finance and Stochastics, Vol. 6, pp. 429-447.

[28] H. Geman and M. Yor (1993) Bessel processes, Asian options and perpertuities. Mathematical Finance, Vol. 3, no 4, pp. 349-375.

[29] J.M. Harisson and S.R. Pliska (1981) Martingales and stochastic integrals in the theory of continuous trading. The Stochastic Processes and Their Applications, Vol. 11, pp. 261-271.

[30] E. Jouiny and H. Kallal (2001) Efficient Trading Strategies in the Presence of Market Frictions. Review of Financial Studies, Vol. 14, pp. 343-69.

[31] D.O. Kramkov (1996) Optional decomposition of supermartingales and hedging contingent claims in incomplete security markets. Probability Theory and Related Fields, Vol. 105, pp. 459-479.

[32] R. Lagnado and S. Osher (1997): A technique for calibrating derivative security pricing models: numerical solution of an inverse problem. The Journal of Computational Finance, Vol. 1, no 1, pp. 13-25.

[33] J.P. Laurent and H. Pham (1999) Dynamic programming and mean-variance hedging. Finance and Stochastics, Vol. 23, pp. 83-110.

[34] P.-L. Lions, N. Touzi, J.-M. Lasry, J. Lebuchoux and E. Fournié (1999) Some applications of Malliavin calculus to Monte Carlo methods in finance. Finance and Stochastics, Vol. 3, pp. 391-412.

[35] P.-L. Lions, J.-M. Lasry, J. Lebuchoux and E. Fournié (2001) Applications of Malliavin calculus to MonteCarlo methods in finance II. Finance and Stochastics, Vol. 5, pp. 201-236.

[36] R. Merton (2001) Future Possibilities in Finance Theory and Finance Practice Selected papers of Bachelier Congress 2000, pp. 47-72. Editors Geman \& alii, Springer.

[37] M. Musiela and T. Zariphopoulou (2001) Pricing and risk management of derivatives written on non-traded assets. Working Paper (http://www.qgroup.org.au/SFMW/musiela.pdf). 
[38] R. Rouge and N. El Karoui (2000) Pricing via utility maximization and entropy. Mathematical Finance, Vol. 10, no. 2, pp. 259-276.

[39] H. Soner, H. Mete and N. Touzi (2000) Superreplication under gamma constraints. SIAM J. Control Optim, Vol. 39, no. 1, pp. 73-96. 